\title{
Comparison of the Effects of Intratympanic Steroid Injection at Different Intervals in Sudden Sensorineural Hearing Loss
}

\author{
Han Kyung Sung, Ju Chang Kang, Kyu Ha Shin, and Yun Suk An \\ Department of Otorhinolaryngology-Head and Neck Surgery, Bundang Jesaeng Hospital, Daejin Medical Center, \\ Seongnam, Korea
}

\author{
Received July 31,2019 \\ Revised September 10, 2019 \\ Accepted October 4, 2019 \\ Address for correspondence \\ Yun Suk An, MD, PhD \\ Department of Otorhinolaryngology- \\ Head and Neck Surgery, \\ Bundang Jesaeng Hospital, \\ Daejin Medical Center, \\ 20 Seohyeon-ro 180beon-gil, \\ Bundang-gu, Seongnam 13590, Korea \\ Tel +82-31-779-0258 \\ Fax +82-31-779-0265 \\ E-mail stella23@dmc.or.kr
}

\begin{abstract}
Background and Objectives: Systemic steroid therapy (SST) and intratympanic steroid injection (ISI) have been the treatment of choice for sudden sensorineural hearing loss (SSNHL). We studied the effect of ISI administered at different intervals on hearing outcomes in patients with SSNHL. Subjects and Methods: We performed a retrospective study of 427 patients diagnosed with SSNHL at Bundang Jesaeng Hospital, of whom 51 patients with SSNHL who received SST and four ISIs were included in this study. Patients were treated with four ISIs either every day for 4 days (group 1) or at intervals (mean duration of interval: 2.21 days) (group 2). Hearing outcomes were evaluated using the pure-tone test before the injection and 14 days, 1 month, and 3 months after the final injection. Recovery rates were classified based on Siegel's criteria. Results: The amount of improvement was $27.67 \mathrm{~dB}( \pm 20.45)$ in group 1 and $32.79 \mathrm{~dB}( \pm 21.42)$ in group 2. However, there were no significant differences between the two groups $(p=0.714)$. The recovery rates based on Siegel's criteria were $18 / 27(66.7 \%)$ and $16 / 24(66.7 \%)$ in groups 1 and 2 , respectively, with no significant difference $(p=1.000)$. Considering only complete recoveries in hearing recovery, the recovery rates were 15/27 (55.6\%) and 14/24 (58.3\%) in groups 1 and 2, respectively, with no significant difference $(p=0.842)$. Conclusions: There were no significant differences in hearing outcomes or recovery rates after ISI administration every day or at intervals of 2-3 days. J Audiol Otol 2020;24(1):24-28
\end{abstract}

KEY WORDS: Correction of hearing loss · Intratympanic injection · Sensorineural hearing loss.

\section{Introduction}

Sudden sensorineural hearing loss can be defined as sudden loss of more than $30 \mathrm{~dB}$ of sensorineural hearing at three or more consecutive frequencies within 3 days [1], and it is treated as an otologic emergency, as failure to receive a proper treatment in the early stage may result in permanent hearing loss [2]. Although the exact mechanism of action for the cause of sudden sensorineural hearing loss is unknown, various etiological theories consisting of various factors have been proposed. While viral infection, vascular disorders, autoimmune diseases, trauma, inner ear deformation, and central nervous system disorders, such as acoustic tumor, are believed to be involved, the cause is often unknown in many patients. Car-

This is an Open Access article distributed under the terms of the Creative Commons Attribution Non-Commercial License (https://creativecommons.org/licenses/by-nc/4.0/) which permits unrestricted non-commercial use, distribution, and reproduction in any medium, provided the original work is properly cited. diovascular diseases, smoking habit, and excessive stress appear to be the most common risk factors associated with sudden sensorineural hearing loss [1].

Therefore, various treatment methods have been tried for sudden sensorineural hearing loss. Systemic steroid therapy is recognized as the basic treatment for sudden sensorineural hearing loss owing to its high anti-inflammatory effect [3], and other known treatments include intratympanic steroid injection, anti-viral agents, diuretics, blood circulation improvement agents, and high-pressure oxygen therapy. Among these, intratympanic steroid injection can be used for patients who are unresponsive to treatment or have difficulty in undergoing the whole-body therapy. It has a significantly higher recovery rate when conducted in parallel with systemic steroid therapy [4], and injecting steroids into the tympanic cavity can help maintain a higher concentration of steroids in the inner ear [5].

Even with these advantages, clear guidelines for treatment 
with intratympanic steroid injection have not established, and there are only a few studies on the treatment effect of intratympanic steroid injection at different intervals. In the study by Chou, et al. [6], there were no statistically significant differences between the treatment effect of the near-continual transtympanic steroid perfusion and the intermittent intratympanic steroid injection. However, the group with the near-continual transtympanic steroid perfusion had better improvement in hearing.

Therefore, under the assumption that intratympanic steroid injections at a shorter interval would result in higher concentrations of steroid inside the inner ear and provide better treatment effect, the aim of this study was to compare the treatment effects of the intratympanic steroid injection at different treatment intervals for patients with sudden sensorineural hearing loss.

\section{Subjects and Methods}

\section{Subjects}

From January 2015 to February 2019, 427 patients were diagnosed with sudden sensorineural hearing loss in our department and treated with systemic steroid therapy and intratympanic steroid injection. Among them, the medical records of 99 patients who received intratympanic steroid injection four times were retrospectively analyzed. Finally, 51 patients were included in this study, excluding the patients whose hearing loss started more than 14 days before, who had less than $25 \mathrm{~dB}$ in the initial hearing test, who had recurrent hearing loss or trauma, and who were lost to follow-up. The initial hearing and presence of dizziness, tinnitus, hypertension, and diabetes were investigated. Further, the results of hearing improvement after the steroid therapy was investigated. The study was approved by the Institutional Review Board of Bundang Jesaeng Hospital (IRB No. DMC 2019-07-003).

\section{Methods}

For systemic steroid therapy, the patients were orally or intravenously injected with $0.8 \mathrm{mg} / \mathrm{kg}$ of methylprednisolone (Methylon $^{\circledR}, 4 \mathrm{mg} / \mathrm{T}$, Alvogen, Gonju, Korea) or $0.15 \mathrm{mg} / \mathrm{kg}$ of dexamethasone disodium phosphate (Dexamethasone ${ }^{\circledR}, 5$ $\mathrm{mg} / \mathrm{mL}$, Yuhan, Seoul, Korea) for 5 days, and subsequently, the dose was reduced over 5 days. The patients underwent systemic steroid treatment using oral steroids. However, if a patient complained of gastrointestinal trouble or difficulties in oral administration, the systemic steroid treatment was provided via the intravenous route. Furthermore, all patients received $160 \mathrm{mg}$ of ginkgo biloba extract (Tanamine, $80 \mathrm{mg} / \mathrm{T}$, Yuyu Pharma Inc., Jecheon, Korea) orally in the follow-up period.

For the intratympanic steroid injection, the patients were injected with $0.4-0.6 \mathrm{~mL}$ of dexamethasone disodium phosphate (Dexamethasone ${ }^{\circledR}, 5 \mathrm{mg} / \mathrm{mL}$, Yuhan). Depending on the interval of the intratympanic steroid injection, the patients who received administrations at intervals of 1 day were assigned into group 1, and patients who received administrations at intervals of 2-3 days were assigned into group 2 .

Pure-tone audiometry was conducted on the day of the hospital visit, after 2 weeks, after 1 month, after 3 months, and in the follow-up period. The patients with fully recovered hearing were eliminated from the study. The results of pure-tone audiometry were calculated in a quartering method of $0.5,1$, and $2 \mathrm{kHz}$, and the degree of initial hearing loss was classified as mild $(26-40 \mathrm{~dB})$, moderate $(41-55 \mathrm{~dB})$, moderate-severe $(56-70 \mathrm{~dB})$, severe $(71-90 \mathrm{~dB})$, and profound $(91 \mathrm{~dB})$. The level of hearing recovery after the treatment was classified based on Siegel's criteria: 1) Complete recovery was defined as the final hearing level of over $25 \mathrm{~dB}$, regardless of the size of the gain; 2) Partial recovery was defined as $\geq 15 \mathrm{~dB}$ of gain and the final hearing level of $25-45 \mathrm{~dB}$; 3) Slight improvement was defined as $\geq 15 \mathrm{~dB}$ of gain and the final hearing level lower than $45 \mathrm{~dB}$; 4) No improvement was defined as $<15 \mathrm{~dB}$ of gain or the final hearing level lower than $75 \mathrm{~dB}$ [7].

\section{Intratympanic steroid injection}

For intratympanic steroid injection, the patient was placed in the supine position. Beracaine spray (Lidocaine, $0.1 \mathrm{~g} / \mathrm{mL}$, Firson Co., Ltd., Cheonan, Korea) was used to induce local anesthesia in the tympanum, and $0.4-0.6 \mathrm{~mL}$ of dexamethasone was injected in the anterosuperior part of the tympanum using a 1-mL syringe with a 25-gauge spinal needle under the microscope. Subsequently, to allow the injected drug to stay in the round window as long as possible, the patient was maintained in the supine position with the symptomatic ear facing upward for $10 \mathrm{~min}$ and instructed not to swallow or speak.

\section{Statistical analysis}

For statistical analyses, the two groups were compared using the t-test, Mann-Whitney U test, chi-square test, and analysis of variance on SPSS 20.0 (IBM Corp., Armonk, NY, USA). A $p$-value below 0.05 was considered statistically significant.

\section{Results}

Group 1 had a total of 27 patients aged $58.48 \pm 15.15$ years, with male-to-female ratio of 9:18. Their hearing capacity was $58.89 \pm 25.81 \mathrm{~dB}$ at the time of occurrence, and the systemic steroid therapy and intratympanic steroid injection treatment were commenced $3.25 \pm 3.32$ days after the onset of hearing 
loss. Among them, 17 patients underwent the systemic steroid therapy via the oral route, and the remaining 10 patients underwent the systemic steroid therapy via the intravenous route. Group 2 had a total of 24 patients aged $58.75 \pm 8.48$ years, with male-to-female ratio of 12:12. Their hearing capacity was $64.06 \pm 32.23 \mathrm{~dB}$ at the time of occurrence, and the systemic steroid therapy and intratympanic steroid injection treatment were commenced $4.33 \pm 4.50$ days after the onset of hearing loss. Among them, 22 patients underwent the systemic steroid therapy via the oral route, and the remaining 2 patients underwent the systemic steroid therapy via the intravenous route. There were no statistically significant differences between the two groups in age, sex ratio, initial hearing level, dizziness, tinnitus, or underlying diseases. The interval for intratympanic steroid injection in group 2 was $2.21 \pm 1.05$ days (Table 1).

Two weeks after the treatment, compared to the initial hearing, groups 1 and 2 had hearing improvements of $25.03 \pm$ $20.36 \mathrm{~dB}$ and $25.50 \pm 26.07 \mathrm{~dB}$, respectively. In the final hearing test in group 1, the average hearing test follow-up period was 42.96 days, with hearing improvement of $27.67 \pm 20.45$ $\mathrm{dB}$ and final pure-tone audiogram of $31.37 \pm 27.48 \mathrm{~dB}$. In group 2, the average hearing test follow-up period was 40.36 days, with hearing improvement of $32.79 \pm 21.42 \mathrm{~dB}$ and final pure-tone audiogram of $31.46 \pm 24.55 \mathrm{~dB}$. The hearing test results showed no statistically significant differences $(p=$
0.714) between the two groups (Fig. 1).

Based on the extent of hearing recovery, group 1 showed a recovery rate of $18 / 27(66.7 \%)$, with 15,3 , and 9 patients showing full recovery, partial recovery, and no recovery, respectively. Group 2 showed a recovery rate of 16/24 (66.7\%), with 14, 2,3 , and 5 patients showing full recovery, partial recovery,

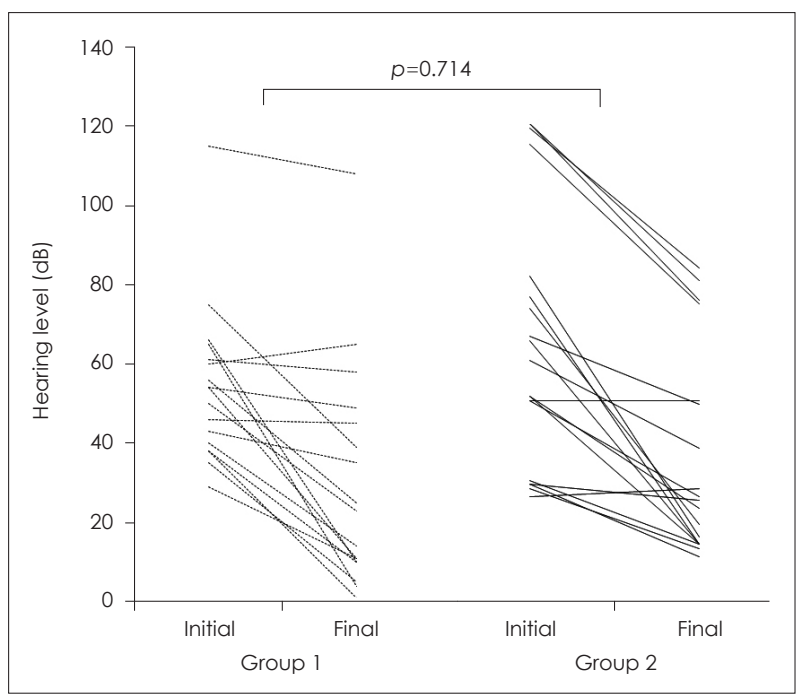

Fig. 1. Comparison of the pure-tone average between the two groups. There were no significant differences in hearing improvement between the two groups ( $p=0.714$, analysis of variance). Group 1: Intratympanic steroid injection every day for 4 days. Group 2: Intratympanic steroid injection four times at intervals of $2-3$ days.

Table 1. Comparison of the demographics and hearing characteristics between the two groups

\begin{tabular}{|c|c|c|c|c|}
\hline Clinical characteristics & Group $1(n=27)$ & Group $2(n=24)$ & Total $(n=51)$ & $p$-value \\
\hline Age (years) & $58.48 \pm 15.15$ & $58.75 \pm 8.48$ & $58.60 \pm 12.30$ & $0.939 *$ \\
\hline Sex & & & & $0.227^{\ddagger}$ \\
\hline Male & $9(33.3)$ & $12(50.0)$ & $21(41.2)$ & \\
\hline Female & $18(66.7)$ & $12(50.0)$ & $30(58.8)$ & \\
\hline Vertigo & $5(18.5)$ & $3(12.5)$ & $8(15.7)$ & $0.555^{\ddagger}$ \\
\hline Tinnitus & $4(14.8)$ & $5(20.8)$ & $9(17.6)$ & $0.574^{\ddagger}$ \\
\hline Vertigo+tinnitus & $2(7.4)$ & $0(0.0)$ & $2(3.9)$ & $0.174^{\ddagger}$ \\
\hline Duration from onset (day) & $3.25 \pm 3.32$ & $4.33 \pm 4.50$ & $3.76 \pm 3.92$ & $0.515^{\dagger}$ \\
\hline Pure tone result ( $\mathrm{dB}$ ) & $58.89 \pm 25.81$ & $64.06 \pm 32.23$ & $61.32 \pm 28.83$ & $0.526^{*}$ \\
\hline Severity of hearing loss (No. of Pt.) & & & & $0.940^{\S}$ \\
\hline Mild & $8(29.6)$ & $7(29.2)$ & $15(29.4)$ & \\
\hline Moderate & $7(25.9)$ & $5(20.8)$ & $12(23.5)$ & \\
\hline Moderate-severe & $5(18.5)$ & $3(12.5)$ & $8(15.7)$ & \\
\hline Severe & $3(11.1)$ & $4(16.7)$ & $7(13.7)$ & \\
\hline Profound & $4(14.8)$ & $5(20.8)$ & $9(17.6)$ & \\
\hline HTN & $7(25.9)$ & $7(29.2)$ & $14(27.5)$ & $0.796^{\ddagger}$ \\
\hline DM & $7(25.9)$ & $5(20.8)$ & $12(23.5)$ & $0.669^{\ddagger}$ \\
\hline IT injection interval (day) & 1 & $2.21 \pm 1.05$ & & \\
\hline
\end{tabular}

Values are presented as meantstandard deviation or $\mathrm{n}(\%)$ unless otherwise indicated. Group 1: Intratympanic steroid injection every day for 4 days, Group 2: Intratympanic steroid injection four times at intervals of 2-3 days. *independent t-test, ${ }^{\dagger}$ MannWhitney U test, ${ }^{\ddagger}$ chi-square independence test, ${ }^{\S}$ Fisher's exact test. n: number, Pt.: patient, HTN: hypertension, DM: diabetes mellitus, dB: decibel 
Table 2. Final hearing recovery rates after intratympanic injections based on Siegel's criteria

\begin{tabular}{lccc}
\hline Siegel's type & Group 1 $(\mathrm{n}=27)$ & Group 2 $(\mathrm{n}=24)$ & $\mathrm{p}$-value \\
\hline Complete & $15(55.6)$ & $14(58.3)$ & 0.842 \\
Partial & $3(11.1)$ & $2(8.3)$ & 0.739 \\
Slight & $0(0.0)$ & $3(12.5)$ & 0.058 \\
No response & $9(33.3)$ & $5(20.8)$ & 0.318 \\
Recovery rate & $18(66.7)$ & $16(66.7)$ & 1.000 \\
\hline
\end{tabular}

Values are presented as $n$ (\%) unless otherwise indicated. Group 1: Intratympanic steroid injection every day for 4 days, Group 2: Intratympanic steroid injection four times at intervals of $2-3$ days. n: number

slight recovery, and no recovery, respectively. The hearing recovery was not statistically different between the two groups $(p=1.000)$ (Table 2). While administering the intratympanic steroid injection over four sessions, no associated complications occurred in either group.

\section{Discussion}

Hobson, et al. [8] reported that a combination of systemic steroid therapy and intratympanic steroid injection as the primary treatment shows better hearing improvement than the systemic steroid therapy or intratympanic steroid injection alone, and they recommended the oral intake of $60 \mathrm{mg} /$ day of prednisone for 7-10 days and four sessions of intratympanic steroid injection with $24 \mathrm{mg} / \mathrm{mL}$ of dexamethasone over 2 weeks. Steroids reach the inner ear via the systemic route or via intratympanic steroid injection and increases blood flow to the inner ear. Systemic steroid therapy requires high concentrations of steroids to provide therapeutic effect because it needs to pass through the blood-labyrinth barrier. In contrast, intratympanic steroid injection requires low concentrations of steroids, as the steroid is directly delivered through the round window [9]. Lee, et al. [10] compared the steroid concentrations in the perilymph when dexamethasone was injected to mice through the round window or through the abdominal cavity and reported that injection through the round window results in higher steroid concentrations and maintains the concentration for a longer period of time. Therefore, despite intratympanic steroid injection being widely used in the clinical treatment of sudden sensorineural hearing loss, various opinions on the optimal dose, medication, and administration methods are still being proposed.

As we thought that administering intratympanic steroid injection at shorter intervals would maintain a higher concentration of steroid within the inner ear and would achieve better treatment effects, we performed this study by dividing the study subjects into two groups: a group that received intratympanic steroid injection every day and another that received intratympanic steroid injection every $2-3$ days. Hargunani, et al. [11] studied the distribution of steroids in the inner ear over time following intratympanic steroid injections in an animal experiment using rats. Steroids were first found in the inner ear $15 \mathrm{~min}$ after the injection and no longer found $24 \mathrm{~h}$ after the injection. There are only a few reports on improvement in the hearing threshold level and the hearing recovery rate based on Siegel's criteria after medication use with the intratympanic steroid injection. Kim, et al. [12] reported no significant differences in the hearing threshold level improvement or hearing recovery rate based on Siegel's criteria with methylprednisolone or dexamethasone injection. However, the authors mentioned that dexamethasone may be the preferred drug, as the use of methylprednisolone causes pain, although without statistical significance, such as burning sensation in the throat and ear [12]. Sugihara, et al. [13] studied the treatment effects based on the interval of intratympanic steroid injection by dividing the patients who received at least two intratympanic steroid injections into three groups (group 1: 1-4day interval, group 2: 5-10-day interval, group 3: 11-30-day interval) and reported that there were no significant differences in the hearing test among different groups and that all groups showed similar hearing results 11-30 days after the treatment. In addition, when Suzuki, et al. [14] compared the results of the hearing tests between the groups that received intratympanic steroid injections at short intervals (4 times a week) and at long intervals (once a week over 4 weeks), there were no statistically significant differences between the two groups.

In this study, systemic steroid therapy and intratympanic steroid injection were simultaneously commenced for an average duration of $3.76 \pm 3.92$ days from the onset of hearing loss. The patients who received a total of four intratympanic steroid injections were divided into two groups: a group that received injection every day and another that received injections every 2-3 days (average 2.21 days). When classifying complete and partial recovery under Siegel's criteria as recovered hearing, both the groups had $66 \%$ of hearing recovery rates, showing no statistically significant differences. In addition, even upon including complete recovery as recovered hearing, the two groups did not show significant differences $(p=0.842)$. 
A limitation of this study was that the steroid types used in the systemic steroid therapy were different between oral methylprednisolone and intravenous dexamethasone therapies. The reason was that if a patient complained of gastrointestinal problems or difficulties in oral administration, the systemic steroid treatment was provided via the intravenous route. In addition, since patients who simultaneously received systemic steroid treatment and intratympanic steroid injection were studied, checking the treatment effect of intratympanic steroid injection alone at different intervals might be limited. Such variables would need to be more controlled in the future studies. Lastly, further studies should be conducted on more patients because the small sample size was a limitation of this study, which had resulted from the excluding nature of the retrospective study.

In conclusion, this study was conducted by dividing the patients with sudden sensorineural hearing loss into a group that received systemic steroid therapy and intratympanic steroid injection every day and another group that received intratympanic steroid injection every $2-3$ days. The results of this study showed no significant differences in the hearing potency or hearing recovery rates between the two groups.

\section{Conflicts of interest}

The authors have no financial conflicts of interest.

\section{Author Contributions}

Conceptualization: Yun Suk An. Data curation: Han Kyung Sung, Ju Chang Kang, and Kyu Ha Shin. Formal analysis: Han Kyung Sung. Writing — original draft: Han Kyung Sung. Writing — review \& editing: Yun Suk An and Han Kyung Sung.

\section{ORCID iDs}

Han Kyung Sung

Ju Chang Kang

Kyu Ha Shin

Yun Suk An https://orcid.org/0000-0001-5847-3546

https://orcid.org/0000-0003-4545-2206

https://orcid.org/0000-0002-3121-6993

https://orcid.org/0000-0002-8570-2938

\section{REFERENCES}

1) Chau JK, Lin JR, Atashband S, Irvine RA, Westerberg BD. Systematic review of the evidence for the etiology of adult sudden sensorineural hearing loss. Laryngoscope 2010;120:1011-21.

2) Vijayendra H, Buggaveeti G, Parikh B, Sangitha R. Sudden sensorineural hearing loss: an otologic emergency. Indian J Otolaryngol Head Neck Surg 2012;64:1-4.

3) Moskowitz D, Lee KJ, Smith HW. Steroid use in idiopathic sudden sensorineural hearing loss. The Laryngoscope 1984;94:664-6.

4) Conlin AE, Parnes LS. Treatment of sudden sensorineural hearing loss: II. A Meta-analysis. Arch Otolaryngol Head Neck Surg 2007; 133:582-6.

5) Parnes LS, Sun AH, Freeman DJ. Corticosteroid pharmacokinetics in the inner ear fluids: an animal study followed by clinical application. Laryngoscope 1999;109(7 Pt 2):1-17.

6) Chou YF, Chen PR, Kuo IJ, Yu SH, Wen YH, Wu HP. Comparison of intermittent intratympanic steroid injection and near-continual transtympanic steroid perfusion as salvage treatments for sudden sensorineural hearing loss. Laryngoscope 2013;123:2264-9.

7) Siegel LG. The treatment of idiopathic sudden sensorineural hearing loss. Otolaryngol Clin North Am 1975;8:467-73.

8) Hobson CE, Alexander TH, Harris JP. Primary treatment of idiopathic sudden sensorineural hearing loss with intratympanic dexamethasone. Curr Opin Otolaryngol Head Neck Surg 2016;24:407-12.

9) Shirwany NA, Seidman MD, Tang W. Effect of transtympanic injection of steroids on cochlear blood flow, auditory sensitivity, and histology in the guinea pig. Am J Otol 1998;19:230-5.

10) Lee JJ, Jang JH, Choo OS, Lim HJ, Choung YH. Steroid intracochlear distribution differs by administration method: systemic versus intratympanic injection. Laryngoscope 2018;128:189-94.

11) Hargunani CA, Kempton JB, DeGagne JM, Trune DR. Intratympanic injection of dexamethasone: time course of inner ear distribution and conversion to its active form. Otol Neurotol 2006;27:564-9.

12) Kim YJ, Jang SU, Lee HH, Kwon JH. Comparison of the effect of intratympanic steroid injection medications in patients with idiopathic sudden sensorineural hearing loss. Korean J Otorhinolaryngol-Head Neck Surg 2017;60:441-8.

13) Sugihara EM, Evans MA, Neumann M, Babu SC. The effect of intratympanic steroid injection frequency in idiopathic sudden sensorineural hearing loss. Am J Otolaryngol 2018;39:688-92.

14) Suzuki H, Koizumi H, Ohkubo J, Hohchi N, Ikezaki S, Kitamura T. Hearing outcome does not depend on the interval of intratympanic steroid administration in idiopathic sudden sensorineural hearing loss. Eur Arch Otorhinolaryngol 2016;273:3101-7. 